\title{
Digestibilidade do Bagaço de Cana-de-açúcar Tratado com Reagentes Químicos e Pressão de Vapor ${ }^{1}$
}

\section{Ricardo Pereira Manzano², Romualdo Shigueo Fukushima ${ }^{3}$, Jacinta Diva Ferrugem Gomes ${ }^{4}$, Geraldo Garippo ${ }^{5}$}

\begin{abstract}
RESUMO - Com o objetivo de elevar a digestibilidade do bagaço de cana-de-açúcar, este resíduo agro-industrial foi tratado com inúmeros reagentes químicos acompanhados ou não de tratamento físico. Após ensaios preliminares, nos quais diversos agentes deslignificantes foram avaliados, dez tratamentos foram selecionados para serem melhor estudados em ensaios de digestibilidade in vitro da matéria seca e da matéria orgânica. Em seguida, para o ensaio de digestibilidade in vivo, foram feitas quatro dietas à base de: 1 - Bagaço auto-hidrolisado (BAH), pressão de $17 \mathrm{kgf} / \mathrm{cm}^{2}$ por $5 \mathrm{~min} ; 2$ - Bagaço tratado com $4 \% \mathrm{Na}_{2} \mathrm{~S}+6 \% \mathrm{NaOH}$, pressão de 12 $\mathrm{kgf} / \mathrm{cm}^{2}$ por $8 \mathrm{~min} ; 3$ - Bagaço tratado com $2 \% \mathrm{Na}_{2} \mathrm{~S}+3 \% \mathrm{NaOH}$, pressão de $12 \mathrm{kgf} / \mathrm{cm}^{2}$ por 8 min; e 4 - Bagaço tratado com $9 \%$ $\mathrm{H}_{2} \mathrm{O}_{2}+7 \% \mathrm{NaOH}$, a $70^{\circ} \mathrm{C}$ por $8 \mathrm{~min}$. Bagaço tratado com $4 \% \mathrm{Na}_{2} \mathrm{~S}+6 \% \mathrm{NaOH}$ e submetido a $12 \mathrm{kgf} / \mathrm{cm}^{2}$ de pressão apresentou os melhores coeficientes de digestibilidade da matéria seca, matéria orgânica, fibra em detergente neutro e fibra em detergente ácido e o maior valor de nutrientes digestíveis totais. Em seguida, o bagaço tratado com $9 \% \mathrm{H}_{2} \mathrm{O}_{2}+7 \% \mathrm{NaOH}$ a $70^{\circ} \mathrm{C}$ por 8 min apresentou os melhores resultados. Piores resultados foram observados para o bagaço hidrolizado. A melhor digestibilidade de algumas das dietas, particularmente das frações fibrosas, sugere a exiqüibilidade do emprego de menores quantidades de alimento concentrado em dietas à base de bagaço de cana tratado química/fisicamente.
\end{abstract}

Palavras-chave: hidróxido de sódio, nutrição de ruminantes, resíduo agro-industrial, sulfeto de sódio

\section{Digestibility of Sugar Cane Bagasse Treated with Chemical Reagents and Steam Pressure}

\begin{abstract}
In order to increase the sugar cane bagasse digestibility, this agricultural by-product was treated with several chemical reagents with or without physical treatment. After preliminary evaluation, where several delignificant agents were evaluated, ten treatments were selected for more detailed in vitro dry and organic matter disappearance trials. Then, for the in vivo digestibility trial, four sugar cane bagasse based diets were made: 1 - Hydrolyzed sugar cane bagasse, pressure of $17 \mathrm{kgf} / \mathrm{cm}^{2}$ per 5 min; 2 - Bagasse treated with $4 \% \mathrm{Na} 2 \mathrm{~S}$ $+6 \% \mathrm{NaOH}$, pressure of $12 \mathrm{kgf} / \mathrm{cm}^{2}$ for $8 \mathrm{~min} ; 3$ - Bagasse treated with $2 \% \mathrm{Na}_{2} \mathrm{~S}+3 \% \mathrm{NaOH}$, pressure of $12 \mathrm{kgf} / \mathrm{cm}^{2}$ for $8 \mathrm{~min} ; 4-\mathrm{Bagasse}$ treated with $9 \% \mathrm{H}_{2} \mathrm{O}_{2}+7 \% \mathrm{NaOH}$, at $70^{\circ} \mathrm{C}$ for $8 \mathrm{~min}$. The bagasse treated with $4 \% \mathrm{Na}_{2} \mathrm{~S}+6 \% \mathrm{NaOH}$ and pressure of $12 \mathrm{kgf} / \mathrm{cm}^{2}$ for 8 min exhibited the highest coefficients of digestibility for dry matter, organic matter, neutral detergent fiber and acid detergent fiber, as well as the highest total digestible nutrient values. Secondly, the bagasse treated with $9 \% \mathrm{H}_{2} \mathrm{O}_{2}+7 \% \mathrm{NaOH}$, at $70^{\circ} \mathrm{C}$ for 8 min showed the best results. Hydrolyzed sugar cane bagasse presented the worst results. The best digestibility data of some diets, particularly of the fibrous fractions, suggests the possibility of reducing the amount of concentrate meal in chemically/physically treated sugar cane based diets.
\end{abstract}

Key Words: agricultural by-product, ruminant nutrition, sodium hydroxide, sodium sulfide

\section{Introdução}

A cana-de-açúcar (Saccharum officinarum, L.) é uma planta de suma importância para a economia brasileira, tornando-se grande geradora de empregos e de energia via industrialização desta em açúcar e álcool. Como conseqüência do processamento industrial da cana, são produzidos inúmeros resíduos, entre eles o bagaço, responsável por 25 a $30 \%$ em peso da cana moída, ou seja, um resíduo da ordem de 74 a 88 milhões de toneladas de bagaço (NUSSIO, 1993). Existem diversas utilizações para o bagaço, que vai desde a produção de energia térmica à indústria de papel e celulose, passando pela alimentação animal; para este último caso, o bagaço apresenta, no entanto, alguns óbices, representado principalmente pelo seu

\footnotetext{
${ }^{1}$ Pesquisa financiada pela Fundação de Amparo à Pesquisa do Estado de São Paulo (FAPESP) e parte da Dissertação de Mestrado do primeiro autor apresentada à Faculdade de Zootecnia e Engenharia de Alimentos da Universidade de São Paulo (FZEA/USP), Av. Duque de Caxias Norte, 225, CEP 13630-000, Pirassununga, SP.

2 Médico Veterinário, bolsista de Mestrado da FAPESP.

${ }^{3}$ Médico Veterinário, Professor Associado do Departamento de Zootecnia da FZEA/USP; tel.: (19)561-2044. E.mail: rsfukush@usp.br Autor para correspondências.

${ }^{4}$ Zootecnista, Professora Doutora do Departamento de Zootecnia da FZEA/USP.

${ }^{5}$ Discente do curso de Zootecnia da FZEA/USP e bolsista de Iniciação Científica da FAPESP.
} 
alto teor de material lignocelulósico, cuja viabilidade de utilização requer o desenvolvimento de métodos de tratamento que promovam o rompimento da estrutura de sua fração fibrosa, para torná-lo mais digestível (BURGI, 1985). No Brasil, o tratamento com pressão e vapor é o método mais utilizado para elevar o valor nutritivo do bagaço de cana-de-açúcar, tornando-o um volumoso de médio valor nutritivo (BEM, 1991).

RANGNEKAR et al. (1982), MARCOS et al. (1984) e BURGI (1985) trataram bagaço de cana com pressão e vapor e, embora os valores de pressão e tempos de tratamento tenham sido diferentes entre si, observaram incremento na digestibilidade in vitro da matéria seca (DIVMS) em relação ao bagaço in natura.

A combinação entre pressão e álcali foi estudada por MARTIN et al. (1976), que observaram incremento nos valores da DIVMS de 11,37 para $61,66 \%$, quando utilizaram $6 \% \mathrm{NaOH}$ e pressão de $6 \mathrm{kgf} / \mathrm{cm}^{2}$ por $30 \mathrm{~min}$. O emprego concomitante de $4 \% \mathrm{NaOH}$ no bagaço de cana tratado sob pressão e vapor elevou a digestibilidade in vitro, comparada ao material que sofreu apenas tratamento físico, de 41,1 para $46,8 \% ; 35,1$ para $47,3 \%$; e 35,1 para $44,8 \%$ quando as pressões foram de 28,$1 ; 35,2$; e $42,2 \mathrm{kgf} / \mathrm{cm}^{2}$ por $1,5 \mathrm{~min}$, respectivamente (HART et al., 1981).

Alta pressão e, conseqüentemente, elevada temperatura são usualmente requeridas para a melhor utilização dos materiais lignocelulósicos (FAHEY JR. et al., 1993), entretanto, tais condições de tratamento podem levar à produção de furfurais e compostos fenólicos no material tratado, que são substâncias potencialmente tóxicas para os microorganismos ruminais (CHEONG et al., 1974; HART et al., 1981). Combinação de tratamento químico com físico traz como uma das vantagens a possibilidade de se reduzir o nível de pressão e temperatura, situação que poderia reduzir a produção de furfurais e compostos fenólicos. Embora o processo de auto-hidrólise aumente o potencial de degradação do bagaço de cana, a eficácia com que a fibra do mesmo é utilizada é baixa, podendo estar relacionada às condições ruminais desfavoráveis à atividade de bactérias celulolíticas (CASTRO e MACHADO, 1989).

O objetivo do presente experimento foi desenvolver um sistema de tratamento físico-químico que elevasse a digestibilidade do bagaço de cana a níveis superiores aos atingidos pelo tratamento físico, à base de pressão e vapor, possibilitando melhor aproveitamento, pelos animais ruminantes, deste subproduto do setor sucro-alcooleiro.

\section{Material e Métodos}

Os testes preliminares foram conduzidos no Laboratório de Bromatologia da Faculdade de Zootecnia e Engenharia de Alimentos da Universidade de São Paulo (FZEA/USP) e no Laboratório de Química Celulose e Energia do Departamento de Ciências Florestais da Escola Superior de Agricultura "Luiz de Queiroz" da Universidade de São Paulo (ESALQ/USP), com o objetivo de obter informações sobre a melhor combinação entre reagente químico e pressão aplicado ao bagaço e posteriormente avaliado por intermédio de ensaios de digestibilidade in vitro. Os ensaios de digestibilidade foram realizados conforme a descrição de FUKUSHIMA et al. (1991), em que os substratos lignocelulósicos passaram apenas pela fase fermentativa.

Para cada teste, empregaram-se 104,4 g de bagaço (na base seca), imerso em $783 \mathrm{~mL}$ da solução química, de acordo com a combinação de reagentes desejada. Após a mistura, os bagaços permaneceram em repouso por seis horas, sendo a seguir tratados com pressão e temperatura em um digestor rotativo. $\mathrm{O}$ tratamento térmico foi dividido em duas etapas, sendo a primeira de ascensão da temperatura, a qual foi variável em função da temperatura de platô, exigida para cada tratamento, e uma segunda etapa, uma vez atingida a temperatura ideal, mantida constante por uma hora, para todos os tratamentos. As temperaturas de platô para todos os tratamentos envolvendo o $\mathrm{Na}_{2} \mathrm{SO}_{3}$ (sulfito de sódio) e o $\mathrm{NaHSO}_{3}$ (bissulfito de sódio) foram de $135^{\circ} \mathrm{C}$; para os tratamentos do tipo sulfato alcalino $\left(\mathrm{Na}_{2} \mathrm{~S}+\mathrm{NaOH}\right.$ e $\left.\mathrm{Na}_{2} \mathrm{~S}+\mathrm{NH}_{4} \mathrm{OH}\right)-$ embora o reagente original seja o sulfeto de sódio, o nome usual do processo é "sulfato alcalino", uma vez que o sulfeto é oxidado para sulfato durante o procedimento - a temperatura foi $170^{\circ} \mathrm{C}$, de acordo com as recomendações de ASSUMPÇÃO (1988). Para os tratamentos com peróxido de hidrogênio $\left(\mathrm{H}_{2} \mathrm{O}_{2}\right)$ em solução alcalina, a temperatura foi de $70^{\circ} \mathrm{C}$ (DANILAS, 1988). Quando a temperatura de platô exigida era de $170^{\circ} \mathrm{C}$, o tempo de ascensão foi de 40 min, contra $30 \mathrm{~min}$ para $135^{\circ} \mathrm{C}$ e $15 \min$ para $70^{\circ} \mathrm{C}$. Ao final do tratamento físico, as amostras foram secas por 72 horas, em estufa de ventilação forçada a $65^{\circ} \mathrm{C}$, com posterior moagem em moinho Wiley, munido com peneira de $1 \mathrm{~mm}$, e a seguir acondicionadas em sacos plásticos devidamente identificados para posteriores análises.

Os tratamentos (entre parênteses as concentrações dos reagentes químicos na base da MS do 
1198 Rev. bras. zootec.

bagaço de cana) foram os seguintes:

1 - Bagaço in natura - BIN

2 - Bagaço auto-hidrolisado a $17 \mathrm{kgf} / \mathrm{cm}^{2}$ por 5 min - BAH

3 - Solução a $0,53 \%$ de $\mathrm{Na}_{2} \mathrm{~S}+0,8 \%$ de $\mathrm{NaOH}$ $\left(4 \% \mathrm{Na}_{2} \mathrm{~S}+6 \% \mathrm{NaOH}\right)$

4 - Solução a $0,4 \%$ de $\mathrm{Na}_{2} \mathrm{~S}+0,6 \%$ de $\mathrm{NaOH}$

$\left(3 \% \mathrm{Na}_{2} \mathrm{~S}+4,5 \% \mathrm{NaOH}\right)$

5 - Solução a $0,27 \%$ de $\mathrm{Na}_{2} \mathrm{~S}+0,4 \%$ de $\mathrm{NaOH}$

$\left(2 \% \mathrm{Na}_{2} \mathrm{~S}+3 \% \mathrm{NaOH}\right)$

6 - Solução a $0,4 \%$ de $\mathrm{Na}_{2} \mathrm{~S}+0,6 \%$ de $\mathrm{NH}_{4} \mathrm{OH}$ $\left(3 \% \mathrm{Na}_{2} \mathrm{~S}+4,5 \% \mathrm{NH}_{4} \mathrm{OH}\right)$

7 - Solução a $1,2 \%$ de $\mathrm{H}_{2} \mathrm{O}_{2}+0,93 \%$ de $\mathrm{NaOH}$ $\left(9 \% \mathrm{H}_{2} \mathrm{O}_{2}+7 \% \mathrm{NaOH}\right)$

8 - Solução a $0,66 \%$ de $\quad \mathrm{Na}_{2} \mathrm{SO}_{3}$ $\left(5 \% \mathrm{Na}_{2} \mathrm{SO}_{3}\right)$

9 - Solução a $0,66 \%$ de $\mathrm{Na}_{2} \mathrm{SO}_{3}+0,33 \%$ de $\mathrm{NH}_{4} \mathrm{OH}\left(5 \% \mathrm{Na}_{2} \mathrm{SO}_{3}+2,5 \% \mathrm{NH}_{4} \mathrm{OH}\right)$

10 - Solução a $0,66 \%$ de $\mathrm{NaHSO}_{3}+0,33 \%$ de $\mathrm{NH}_{4} \mathrm{OH}\left(5 \% \mathrm{NaHSO}_{3}+2,5 \% \mathrm{NH}_{4} \mathrm{OH}\right)$

Os dados foram analisados com o auxílio do pacote estatístico SAS. No experimento de digestibilidade in vitro, as médias foram comparadas utilizando-se o teste "t" de Student (STEEL e TORRIE, 1980), considerando o nível de significância de 5\%. Foram efetuadas as seguintes comparações:

* Média do tratamento 1 (BIN) versus média do tratamento 2 (BAH).

*Média dos tratamentos 1 e 2 (BIN e BAH) versus média dos tratamentos 3 a 10 .

* Média dos tratamentos 3 , 4 e $5\left(4 \% \mathrm{Na}_{2} \mathrm{~S}+6 \%\right.$ $\mathrm{NaOH} ; 3 \% \mathrm{Na}_{2} \mathrm{~S}+4,5 \% \mathrm{NaOH} ; 2 \% \mathrm{Na}_{2} \mathrm{~S}+3 \%$ $\mathrm{NaOH})$ versus média do tratamento $6\left(3 \% \mathrm{Na}_{2} \mathrm{~S}+\right.$ $\left.4,5 \% \mathrm{NH}_{4} \mathrm{OH}\right)$.

* Média do tratamento $4\left(3 \% \mathrm{Na}_{2} \mathrm{~S}+4,5 \%\right.$ $\mathrm{NaOH})$ versus média do tratamento $6\left(3 \% \mathrm{Na}_{2} \mathrm{~S}+\right.$ $4,5 \% \mathrm{NH}_{4} \mathrm{OH}$ ).

* Média do tratamento $3\left(4 \% \mathrm{Na}_{2} \mathrm{~S}+6 \%\right.$ $\mathrm{NaOH})$ versus média do tratamento $7(9 \%$ de $\mathrm{H}_{2} \mathrm{O}_{2}+7 \%$ de $\mathrm{NaOH}$ ).

*Média do tratamento $8\left(5 \% \mathrm{NaSO}_{3}\right)$ versus média do tratamento $9\left(5 \% \mathrm{NaSO}_{3}+2,5 \% \mathrm{NH}_{4} \mathrm{OH}\right)$.

* Média do tratamento $9\left(5 \% \mathrm{NaSO}_{3}+2,5 \%\right.$ $\left.\mathrm{NH}_{4} \mathrm{OH}\right)$ versus média do tratamento $10(5 \%$ $\left.\mathrm{NaHSO}_{3}+2,5 \% \mathrm{NH}_{4} \mathrm{OH}\right)$.

* Média do tratamento $3\left(4 \% \mathrm{Na}_{2} \mathrm{~S}+6 \% \mathrm{NaOH}\right)$ versus média dos tratamentos 4 e $5\left(3 \% \mathrm{Na}_{2} \mathrm{~S}+4,5 \%\right.$ $\left.\mathrm{NaOH} ; 2 \% \mathrm{Na}_{2} \mathrm{~S}+3 \% \mathrm{NaOH}\right) / 2$.

*Média do tratamento $4\left(3 \% \mathrm{Na}_{2} \mathrm{~S}+4,5 \% \mathrm{NaOH}\right)$ versus média do tratamento $5\left(2 \% \mathrm{Na}_{2} \mathrm{~S}+3 \% \mathrm{NaOH}\right)$.

Frente aos resultados obtidos no ensaio de digestibilidade in vitro, quatro tratamentos foram selecionados para serem tratados industrialmente e, a seguir, confeccionadas quatro rações para serem fornecidas a ovinos em um ensaio de digestibilidade aparente. O processamento industrial do bagaço foi realizado na Usina Santa Luíza, município de Matão-SP, a qual dispõe de um equipamento de hidrólise. Foram pesados $400 \mathrm{~kg}$ (equivalente a $200 \mathrm{~kg}$ de MS) de bagaço e colocados em um vagão misturador de rações, equipado com três roscas-sem-fim; sobre o bagaço foram aspergidos $400 \mathrm{~L}$ de solução de acordo com o tratamento, objetivando atingir as concentrações dos reagentes em relação à matéria seca, com concomitante mistura do material. O tempo total deste procedimento foi de 20 minutos. Ao final desta operação, foram separados $400 \mathrm{~kg}$ da mistura (aproximadamente $100 \mathrm{~kg}$ de MS), com o restante sendo desprezado, para se proceder à hidrólise deste material. A pressão e a temperatura do processo de hidrólise foram variadas de acordo com o tratamento químico previamente efetuado, sendo a pressão determinada em função da temperatura requerida para os processos sulfato e peróxido. Os tratamentos estão relatados a seguir:

Tratamento 1 - BAH a $17 \mathrm{kgf} / \mathrm{cm}^{2}$ por $5 \mathrm{~min}$ (não houve "período de ascensão" da temperatura, sendo a injeção de vapor rápida, cerca de $1 \mathrm{~min}$, e a temperatura de platô de 180 a $190^{\circ} \mathrm{C}$ ).

Tratamento 2 - Bagaço tratado com $4 \% \mathrm{Na}_{2} \mathrm{~S}+$ $6 \% \mathrm{NaOH}$ a $12 \mathrm{kgf} / \mathrm{cm}^{2}$ por 8 min (o período de ascensão da temperatura foi de $15 \mathrm{~min}$, com injeções de vapor somando $3 \mathrm{kgf} / \mathrm{cm}^{2}$ a cada $5 \mathrm{~min}$, aproximadamente, e temperatura de platô de $160^{\circ} \mathrm{C}$ ).

Tratamento 3 - Bagaço tratado com $2 \% \mathrm{Na}_{2} \mathrm{~S}+$ $3 \% \mathrm{NaOH}$ a $12 \mathrm{kgf} / \mathrm{cm}^{2}$ por $8 \mathrm{~min}$ (o período de ascensão da temperatura foi de $15 \mathrm{~min}$, com injeções de vapor somando $3 \mathrm{kgf} / \mathrm{cm}^{2}$ a cada $5 \mathrm{~min}$, aproximadamente, e temperatura de platô de $160^{\circ} \mathrm{C}$ ).

Tratamento 4 - Bagaço tratado com $9 \% \mathrm{H}_{2} \mathrm{O}_{2}+$ $7 \% \mathrm{NaOH}$ por 8 min. Neste tratamento, a temperatura foi ao redor de $70^{\circ} \mathrm{C}$. Não houve necessidade de injeção de vapor para atingir esta temperatura, e a injeção de vapor visou apenas o efeito "explosão" e o esvaziamento do hidrolizador.

O ensaio de digestibilidade aparente com ovinos foi realizado nas dependências da Faculdade de Zootecnia e Engenharia de Alimentos da USP, em Pirassununga-SP. Foram utilizados 16 ovinos (Ovis aries L.) da raça Morada Nova, pesando, em média, $18 \mathrm{~kg}$ no início do experimento, previamente vacinados contra febre aftosa e vermifugados. Durante o 
experimento, um dos animais foi retirado do tratamento controle, pois não apresentava condições razoáveis de saúde para permanecer em experimentação, ficando este tratamento apenas com três repetições. O delineamento experimental utilizado foi o inteiramente casualizado, com quatro tratamentos e quatro repetições (STEEL e TORRIE, 1980).

As rações foram compostas de um dos quatro bagaços tratados mais uma mistura de alimentos protéicos (levedura seca + soja integral tostada), perfazendo uma relação volumoso:concentrado de 75:25. A quantidade de ração fornecida durante o período experimental foi equivalente a $2 \%$ do peso vivo dos animais. As rações foram balanceadas para serem isoprotéicas, de acordo com as exigências estabelecidas pelo NATIONAL RESEARCH COUNCIL - NRC (1985), para ovinos em manutenção. A composição bromatológica dos bagaços constituintes das rações (incluindo-se também o bagaço in natura) e a proporção dos ingredientes nas rações constam das Tabelas 1 e 2, respectivamente.

O fornecimento das rações era feito duas vezes ao dia, às 7 e 16 h, representando a ingestão voluntária média dos animais, durante o período de adaptação.
Utilizou-se o método da coleta total de fezes. Durante o período experimental, os animais permaneceram em gaiolas metálicas de digestibilidade, equipadas com cochos e bebedouros. Estas gaiolas foram alojadas em um galpão coberto de alvenaria.

A coleta de fezes foi realizada uma vez ao dia, às 6 h, com pesagem e separação de uma alíquota, referente a $50 \%$ do peso total coletado, para posteriores análises bromatológicas. Matéria seca, matéria orgânica, proteína bruta, extrato etéreo e matéria mineral foram determinados conforme as especificações da ASSOCIATION OF OFFICIAL ANALYTICAL CHEMISTS - AOAC (1990). As frações fibra em detergente neutro (FDN) e fibra em detergente ácido (FDA) foram determinadas conforme as metodologias descritas por GOERING e VAN SOEST (1970). A lignina foi determinada pelo método da lignina em detergente ácido (VAN SOEST, 1963).

As comparações entre os resultados das análises bromatológicas das quatro rações e os coeficientes de digestibilidade aparente obtidos no ensaio com ovinos foram realizadas por intermédio do teste Tukey (STEEL e TORRIE, 1980), considerando-se nível de significância de $5 \%$.

Tabela 1 - Composição bromatológica do bagaço in natura e dos bagaços utilizados no experimento de digestibilidade in vivo ${ }^{1}$ Table 1 - Chemical composition of the in natura bagasse and the bagasses used in the in vivo digestibility trial ${ }^{1}$

\begin{tabular}{|c|c|c|c|c|c|}
\hline $\begin{array}{l}\text { Nutriente } \\
\text { Nutrient }\end{array}$ & $\begin{array}{l}\mathrm{BIN} \\
I N B\end{array}$ & $\begin{array}{c}\mathrm{BAH} \\
H S C B\end{array}$ & $\begin{array}{l}\text { Bagaço sulfato } 1 \\
\text { Sulfate } 1 \text { bagasse }\end{array}$ & $\begin{array}{l}\text { Bagaço sulfato } 2 \\
\text { Sulfate } 2 \text { bagasse }\end{array}$ & $\begin{array}{l}\text { Bagaço peróxido } \\
\text { Peroxide bagasse }\end{array}$ \\
\hline $\begin{array}{l}\text { Proteína bruta } \\
\text { Crude protein }\end{array}$ & $0,8^{\mathrm{A}}$ & $0,8^{\mathrm{A}}$ & $0,4^{\mathrm{B}}$ & $0,4^{\mathrm{B}}$ & $0,5^{\mathrm{A}, \mathrm{B}}$ \\
\hline $\begin{array}{l}\text { Extrato não-nitrogenado } \\
\text { Nitrogen free extract }\end{array}$ & 54,9 & 59,6 & 53,4 & 55,4 & 53,6 \\
\hline $\begin{array}{l}\text { Extrato etéreo } \\
\text { Ether extract }\end{array}$ & 0,6 & 0,8 & 0,7 & 0,8 & 0,6 \\
\hline $\begin{array}{l}\text { Fibra em detergente neutro } \\
\text { Neutral detergent fiber }\end{array}$ & $88,3^{\mathrm{A}}$ & $61,3^{\mathrm{C}}$ & $60,4^{\mathrm{C}}$ & $72,1^{\mathrm{B}}$ & $66,1^{\mathrm{B}, \mathrm{C}}$ \\
\hline $\begin{array}{l}\text { Fibra em detergente ácido } \\
\text { Acid detergent fiber }\end{array}$ & 54,4 & 51,8 & 51,0 & 56,7 & 54,2 \\
\hline $\begin{array}{l}\text { Celulose } \\
\text { Cellulose }\end{array}$ & 41,7 & 41,2 & 44,2 & 44,7 & 44,9 \\
\hline $\begin{array}{l}\text { Hemicelulose } \\
\text { Hemicellulose }\end{array}$ & $34,0^{\mathrm{A}}$ & $9,4^{\mathrm{B}}$ & $9,4^{\mathrm{B}}$ & $15,4^{\mathrm{B}}$ & $11,9^{\mathrm{B}}$ \\
\hline $\begin{array}{l}\text { Lignina em detergente ácido } \\
\text { Acid detergent lignin }\end{array}$ & $12,6^{\mathrm{A}}$ & $10,6^{\mathrm{A}, \mathrm{B}}$ & $6,7^{\mathrm{B}}$ & $12,0^{\mathrm{A}, \mathrm{B}}$ & $9,2^{\mathrm{A}, \mathrm{B}}$ \\
\hline $\begin{array}{l}\text { Matéria mineral } \\
\text { Mineral matter }\end{array}$ & $1,4^{\mathrm{C}}$ & $1,4^{\mathrm{C}}$ & $4,5^{\mathrm{A}}$ & $2,8^{\mathrm{B}, \mathrm{C}}$ & $3,5^{\mathrm{A}, \mathrm{B}}$ \\
\hline
\end{tabular}


1200 Rev. bras. zootec.

Tabela 2 - Proporção dos ingredientes nas rações experimentais (\% da matéria seca)

Table 2 - Proportion of ingredients in the experimental diets (\% dry matter)

\begin{tabular}{lcccc}
\hline $\begin{array}{l}\text { Ingrediente } \\
\text { Ingredient }\end{array}$ & \multicolumn{4}{c}{$\begin{array}{c}\text { Dieta } \\
\text { Diet }\end{array}$} \\
\cline { 2 - 5 } & 1 & 2 & 3 & 4 \\
\hline BAH & 75,0 & - & - & - \\
$\begin{array}{l}\text { HSCB } \\
\text { Bagaço sulfato 1 }\end{array}$ & - & 75,0 & - & - \\
$\begin{array}{l}\text { Sulfate 1 bagasse } \\
\text { Bagaço sulfato 2 }\end{array}$ & - & - & 75,0 & - \\
$\begin{array}{l}\text { Sulfate 2 bagasse } \\
\text { Bagaço peróxido } \\
\begin{array}{l}\text { Peroxide bagasse } \\
\text { Levedura seca }\end{array}\end{array}$ & - & - & - & 75,0 \\
$\begin{array}{l}\text { Dried yeast } \\
\text { Grão de soja tostado }\end{array}$ & 12,0 & 12,0 & 12,0 & 12,0 \\
$\begin{array}{l}\text { Roasted soybean } \\
\text { Mistura mineral } \\
\text { Mineral mixture }\end{array}$ & 1,0 & 1,0 & 12,0 & 12,0 \\
\hline
\end{tabular}

${ }^{1} \mathrm{BAH}=$ bagaço tratado com pressão e vapor; Bagaço sulfato 1 = bagaço tratado com $4 \% \mathrm{Na}_{2} \mathrm{~S}+6 \% \mathrm{NaOH}$; Bagaço sulfato 2 = bagaço tratado com $2 \% \mathrm{Na}_{2} \mathrm{~S}+3 \% \mathrm{NaOH}$; Bagaço peróxido = bagaço tratado com $9 \% \mathrm{H}_{2} \mathrm{O}_{2}+7 \% \mathrm{NaOH}$.

${ }^{1}$ HSCB = Hydrolyzed sugar cane bagasse; Sulfate 1 bagasse = bagasse treated with $4 \% \mathrm{Na}_{2} \mathrm{~S}+6 \% \mathrm{NaOH}$; Sulfate 2 bagasse = bagasse treated with $2 \% \mathrm{Na}_{2} \mathrm{~S}+3 \% \mathrm{NaOH}$; Peroxide bagasse = bagasse treated with $9 \%$ $\mathrm{H}_{2} \mathrm{O}_{2}+7 \% \mathrm{NaOH}$

\section{Resultados e Discussão}

Os resultados da digestibilidade in vitro da matéria seca (DIVMS) e da matéria orgânica (DIVMO) podem ser visualizados na Tabela 3 . O valor da DIVMS do BIN, de $32,4 \%$, foi semelhante ao obtido por BURGI (1985), de 33,3\%, sustentando o fato de o bagaço de cana ser um material lignocelulósico de baixo valor nutritivo, primordialmente pela sua baixa digestibilidade (BOIN et al. 1987).

Os valores da DIVMS e DIVMO dos bagaços tratados (tratamentos 3 a 10) foram superiores $(\mathrm{P}<0,05)$ em relação à média dos tratamentos $\mathrm{BIN}$ e BAH, ou seja, o tratamento do bagaço de cana com compostos químicos sulfurados, em solução alcalina ou não, acoplado ao tratamento com pressão e vapor, e peróxido de hidrogênio alcalino, elevou os valores de digestibilidade in vitro da MS e MO do bagaço de cana. Não houve diferenças entre o BIN e o BAH. Foi constatado valor de DIVMS inferior, apresentado pelo BAH no presente experimento $(36,9 \%)$, quando confrontado com a literatura. Ressalvando-se possíveis diferenças inerentes quanto às condições de processamento do BAH, RANGNEKAR et al. (1982), MARCOS et al. (1984) e BURGI (1985), ao tratarem o bagaço de cana com $7 \mathrm{kgf} / \mathrm{cm}^{2}$ por $30 \mathrm{~min}, 9 \mathrm{kgf} / \mathrm{cm}^{2}$ por $10 \mathrm{~min}$ e $17 \mathrm{kgf} / \mathrm{cm}^{2}$ por $5 \mathrm{~min}$, encontraram valores de DIVMS de 51,1; 52,2; e 64,8, respectivamente.

Todos os valores de digestibilidade apresentados pelos bagaços tratados com $\mathrm{Na}_{2} \mathrm{~S}+\mathrm{NaOH}$ (tratamentos 3 , 4 e 5) foram diferentes entre si $(\mathrm{P}<0,05) \mathrm{e}$ superiores aos bagaços dos tratamentos controle (BIN e BAH), com destaque para o bagaço do tratamento $3\left(4,0 \% \mathrm{Na}_{2} \mathrm{~S}+6,0 \% \mathrm{NaOH}\right)$, que incrementou a DIVMO em $129 \%$ em relação ao BIN, e em $100 \%$ em relação ao BAH. Apesar de os trabalhos registrados na literatura terem empregado diferentes condições de tratamento, bem como diferentes reagentes e respectivas combinações, algumas comparações podem ser permitidas. O tratamento 3 registrou valor de digestibilidade in vitro para a MS de $62,4 \%$, semelhante ao obtido por MARTIN et al. (1976), que registraram valor de $61,66 \%$ para a DIVMS do bagaço de cana tratado com $6 \% \mathrm{NaOH}$ e $6 \mathrm{kgf} / \mathrm{cm}^{2}$. No tratamento $5(2 \%$ $\mathrm{Na}_{2} \mathrm{~S}+3 \% \mathrm{NaOH}$ ), foi utilizada a metade das concentrações de $\mathrm{Na}_{2} \mathrm{~S}$ e $\mathrm{NaOH}$ em relação ao tratamento 3, observando-se valor de DIVMS de 48,7\%, semelhante ao resultado obtido por HART et al. (1981), de 47,3\%, quando trataram bagaço de cana com $4 \% \mathrm{NaOH}$ e pressão de $35,2 \mathrm{kgf} / \mathrm{cm}^{2}$ por $1,5 \mathrm{~min}$, entretanto, o tratamento $4\left(3,0 \% \mathrm{Na}_{2} \mathrm{~S}+4,5 \% \mathrm{NaOH}\right)$

Tabela 3 - Valores de digestibilidade in vitro da matéria seca e da matéria orgânica do bagaço de cana, conforme os tratamentos selecionados a partir dos testes preliminares ${ }^{1}$

Table 3 - In vitro dry and organic matter disappearance data of sugar cane bagasse according to the treatments selected from preliminary trials ${ }^{1}$

\begin{tabular}{lcc}
\hline $\begin{array}{l}\text { Tratamento }^{2} \\
\text { Treatment }\end{array}$ & $\begin{array}{c}\text { DIVMS (\%) } \\
\text { IVDMD }\end{array}$ & $\begin{array}{c}\text { DIVMO (\%) } \\
\text { IVOMD }\end{array}$ \\
\hline $\begin{array}{l}\text { Padrão (feno de Tânzania) } \\
\text { Control (Tanzania hay) }\end{array}$ & 42,3 & 46,0 \\
1 - BIN & 32,4 & 32,9 \\
1 - INB & & \\
2 - BAH & 36,9 & 37,5 \\
$2-H S C B$ & & \\
$3-4 \% \mathrm{Na}_{2} \mathrm{~S}+6 \% \mathrm{NaOH}$ & 62,4 & 75,4 \\
$4-3 \% \mathrm{Na}_{2} \mathrm{~S}+4,5 \% \mathrm{NaOH}$ & 54,9 & 60,1 \\
$5-2 \% \mathrm{Na}_{2} \mathrm{~S}+3 \% \mathrm{NaOH}$ & 48,7 & 52,1 \\
$6-3 \% \mathrm{Na}_{2} \mathrm{~S}+4,5 \% \mathrm{NH}$ & $\mathrm{OH}$ \\
$7-9 \% \mathrm{H}_{2} \mathrm{O}_{2}+7 \% \mathrm{NaOH}$ & 39,3 & 40,9 \\
$8-5 \% \mathrm{Na}_{2} \mathrm{SO}_{3}$ & 59,7 & 65,6 \\
$9-5 \% \mathrm{Na}_{2} \mathrm{SO}_{3}+2,5 \% \mathrm{NH}_{4} \mathrm{OH}$ & 40,2 & 43,5 \\
$10-5 \% \mathrm{NaHSO}_{3}+2,5 \% \mathrm{NH}_{4} \mathrm{OH}$ & 41,0 & 45,6 \\
\hline
\end{tabular}

${ }^{1}$ Dados são médias de três observações.

${ }^{2} \mathrm{BIN}$ - Bagaço in natura; BAH - Bagaço auto-hidrolisado.

${ }_{1}$ Data are means of three replications.

2 INB = In natura bagasse; $H S C B=$ hydrolyzed sugar cane bagasse . 
mostrou índice superior, de 54,9\%.

$\mathrm{Na}$ comparação entre os tratamentos 4 e 6 , que possuíam as mesmas concentrações de $\mathrm{Na}_{2} \mathrm{~S}$ e de álcali, notou-se superioridade $(\mathrm{P}<0,05)$ do tratamento 4, que utilizou $\mathrm{NaOH}$ como álcali, em contrapartida ao $\mathrm{NH}_{4} \mathrm{OH}$ do tratamento 6. Possivelmente esta superioridade se deve ao fato de o $\mathrm{NaOH}$ ser um álcali mais forte que o $\mathrm{NH}_{4} \mathrm{OH}$. ANDRADE e FUKUSHIMA (1994) trataram bagaço de cana com soluções alcalinas de peróxido de hidrogênio e observaram superioridade do $\mathrm{NaOH}$ em relação ao $\mathrm{NH}_{4} \mathrm{OH}$ em deslignificar o bagaço incrementando a sua digestibilidade. A justificativa para o emprego de álcalis reside no fato de a lignina de gramíneas ser particularmente susceptível ao ataque hidrolítico dos mesmos, nas ligações covalentes do tipo éster entre a lignina e a parede celular (VAN SOEST, 1994), e os compostos sulfurados agem formando o íon sulfeto, que em meio alcalino desestabiliza a molécula de lignina (DANILAS, 1988).

Entre os tratamentos testados neste ensaio, merece também destaque o tratamento $7\left(9 \% \mathrm{H}_{2} \mathrm{O}_{2}+\right.$ $7 \% \mathrm{NaOH}$ ), o qual apresentou valor de DIVMS de $59,7 \%$, semelhante ao tratamento 3 , existindo diferença estatística $(\mathrm{P}<0,05)$ apenas para o valor da DIVMO, favorecendo o processo sulfato alcalino. AMJED et al. (1992) trataram bagaço de cana com peróxido de hidrogênio em solução alcalina e obtiveram incremento da ordem de $166 \%$ na digestibilidade daMS, de 18,8 para $50,0 \%$. O mecanismo de ação proposto para o peróxido de hidrogênioé que o ânion hidroperóxido $\left(\mathrm{HO}_{2}{ }^{-}\right)$, formado quando o peróxido de hidrogênio se dissocia, ataca as unidades fenilpropano da lignina em locais elétron deficientes (FAHEY JR. et al., 1993).

Nos tratamentos em que se empregaram sulfito ou bissulfito de sódio, a análise estatística não demonstrou diferenças entre os bagaços tratados com estas substâncias, com ou sem álcalis. Apesar de terem apresentado valores de digestibilidade superiores $(\mathrm{P}<0,05)$ ao $\mathrm{BIN}$ e $\mathrm{BAH}$, os valores numéricos foram inferiores aos valores de DIVMS e DIVMO dos bagaços tratados pelo processo sulfato alcalino. Possivelmente a maior ação benéfica dos tratamentos físico-químicos tenha sido sobre as frações fibrosas, principais componentes do bagaço de cana, tornandoas mais acessíveis às enzimas microbianas.

A composição bromatológica dos quatro bagaços tratados industrialmente, que foram utilizados para o ensaio de digestibilidade in vivo, mais a do bagaço in natura (BIN), pode ser visualizada na Tabela 1 . As principais alterações ocorreram nas frações $\mathrm{PB}, \mathrm{FDN}$, hemicelulose, lignina e matéria mineral $(\mathrm{P}<0,05)$. $\mathrm{O}$ teor de FDN foi maior para o BIN em relação aos demais bagaços $(\mathrm{P}<0,05)$. A variação observada na fração FDN foi reflexo da variação detectada para a concentração de hemicelulose, que foi significativamente menor para todos os bagaços tratados (BAH e os processados física e quimicamente). Ambos os tratamentos físico (BURGI, 1985) e químico (JACKSON, 1977) degradam apreciáveis quantidades de hemicelulose. Segundo CHEONG et al. (1974), o principal produto final da hidrólise da hemicelulose seria o furfural. Como seria de se esperar, o teor de celulose manteve-se inalterado, pois são raros registros de solubilização desta fração carboidrato, quando materiais lignocelulósicos são submetidos a diversos tipos de tratamentos (JACKSON, 1977). Todos os bagaços tratados mostraram menores valores de lignina relativo ao exibido pelo BIN, sendo, entretanto, detectada diferença estatística $(\mathrm{P}<0,05)$ apenas para o tratamento sulfato alcalino $1\left(4 \% \mathrm{Na}_{2} \mathrm{~S}+6 \%\right.$ $\mathrm{NaOH})$. Reagentes hidrolíticos alcalinos como o hidróxido de sódio provocam solubilização parcial da hemicelulose e lignina, por agirem nas ligações que as unem, covalentes e do tipo ester, que são as encontradas nas gramíneas (VAN SOEST, 1994). A fração FDA não mostrou diferenças significativas entre os diversos bagaços, embora, como descrito anteriormente, tivessem sido detectadas diferenças nos valores de lignina. REXEN (1979) observou redução no conteúdo de FDN, enquanto a FDA e a lignina não apresentaram alterações significativas em vários resíduos de cultura após tratamento químico com álcali. NOLTE et al. (1987), porém, observaram decréscimos nas concentrações de FDN, FDA e lignina na palhada de trigo tratada com $4 \% \mathrm{NaOH}$. As diferenças observadas nas concentrações de matéria mineral são apenas reflexo das quantidades de $\mathrm{Na}_{2} \mathrm{~S}$ e $\mathrm{NaOH}$ empregadas nos tratamentos químicos.

Os valores dos coeficientes de digestibilidade aparente da MS, da MO e dos nutrientes e os valores obtidos para os nutrientes digestíveis totais (NDT) das quatro rações experimentais constam da Tabela 4. A ração contendo o bagaço do tratamento $2\left(4 \% \mathrm{Na}_{2} \mathrm{~S}\right.$ $+6 \% \mathrm{NaOH})$ apresentou coeficiente de digestibilidade da MS (CDMS) superior às demais rações $(\mathrm{P}<0,05)$. A ração contendo bagaço do tratamento $4\left(9 \% \mathrm{H}_{2} \mathrm{O}_{2}\right.$ $+7 \% \mathrm{NaOH}$ ) apresentou CDMS superior ao controle (BAH) e ao tratamento $3\left(2 \% \mathrm{Na}_{2} \mathrm{~S}+3 \% \mathrm{NaOH}\right)$ $(\mathrm{P}<0,05)$. Já a dieta contendo o bagaço do tratamento 3 não apresentou diferença estatística, quando comparada à ração controle. Valores observados para a 
1202 Rev. bras. zootec.

Tabela 4 - Coeficientes de digestibilidade da matéria seca (CDMS), matéria orgânica (CDMO), proteína bruta (CDPB), extrato etéreo (CDEE), extrato nãonitrogenado (CDENN), fibra em detergente neutro (CDFDN) e fibra em detergente ácido (CDFDA), e valor do nutriente digestível total (NDT) das rações experimentais, em porcentagem ${ }^{1}$

Table 4 - Digestibility coefficients for dry matter (DCDM), organic matter (DCOM), crude protein (DCCP), ether extract (DCEE), nitrogen free extract (DCNFE), neutral detergent fiber (DCNDF) and acid detergent fiber (DCADF), and the value for total digestible nutrients (TDN) of the experimental diets, in percentage

\begin{tabular}{|c|c|c|c|c|c|}
\hline \multirow[t]{2}{*}{$\begin{array}{l}\text { Coeficient } \\
\text { Coefficient }\end{array}$} & \multicolumn{4}{|c|}{$\begin{array}{c}\operatorname{Dieta}^{2}(\%) \\
\text { Diet }^{2}\end{array}$} & \multirow[b]{2}{*}{$\mathrm{CV}(\%)$} \\
\hline & 1 & 2 & 3 & 4 & \\
\hline $\begin{array}{l}\text { CDMS } \\
D C D M\end{array}$ & $43,9^{\mathrm{C}}$ & $60,6^{\mathrm{A}}$ & $45,6^{\mathrm{C}}$ & $55,6^{\mathrm{B}}$ & 2,57 \\
\hline $\begin{array}{l}\text { CDMO } \\
\text { DCOM }\end{array}$ & $46,9^{\mathrm{D}}$ & $66,1^{\mathrm{A}}$ & $51,3^{\mathrm{C}}$ & $61,8^{\mathrm{B}}$ & 2,57 \\
\hline $\begin{array}{l}\text { CDPB } \\
D C C P\end{array}$ & $43,3^{\mathrm{A}}$ & $44,1^{\mathrm{A}}$ & $44,3^{\mathrm{A}}$ & $46,8^{\mathrm{A}}$ & 5,90 \\
\hline $\begin{array}{l}\text { CDEE } \\
D C E E\end{array}$ & $79,8^{\mathrm{A}}$ & $77,5^{\mathrm{A}}$ & $74,5^{\mathrm{A}}$ & $77,8^{\mathrm{A}}$ & 7,24 \\
\hline $\begin{array}{l}\text { CDENN } \\
D C N F E\end{array}$ & $50,4^{\mathrm{B}}$ & $64,5^{\mathrm{A}}$ & $53,0^{\mathrm{B}}$ & $65,4^{\mathrm{A}}$ & 4,72 \\
\hline $\begin{array}{l}\text { CDFDA } \\
D C A D F\end{array}$ & $25,5^{\mathrm{D}}$ & $64,1^{\mathrm{A}}$ & $39,9^{\mathrm{C}}$ & $48,6^{\mathrm{B}}$ & 7,90 \\
\hline $\begin{array}{l}\text { CDFDN } \\
D C N D F\end{array}$ & $21,1^{\mathrm{D}}$ & $48,9^{\mathrm{A}}$ & $29,8^{\mathrm{C}}$ & $39,6^{\mathrm{B}}$ & 4,11 \\
\hline $\begin{array}{l}\text { NDT } \\
T D N\end{array}$ & $47,2^{\mathrm{C}}$ & $64,3^{\mathrm{A}}$ & $50,6^{\mathrm{C}}$ & $60,6^{\mathrm{B}}$ & 3,03 \\
\hline
\end{tabular}

${ }^{1}$ Médias seguidas por diferentes letras na mesma linha diferem pelo teste Tukey $(p<0,05)$.

$21=\mathrm{BAH}$ (bagaço auto-hidrolisado); $2=4 \% \mathrm{Na}_{2} \mathrm{~S}+6 \% \mathrm{NaOH}$; $3=2 \% \mathrm{Na}_{2} \mathrm{~S}+3 \% \mathrm{NaOH} ; 4=9 \% \mathrm{H}_{2} \mathrm{O}_{2}+7 \% \mathrm{NaOH}$.

${ }^{1}$ Means followed by different letters in the same row differ by Tukey test $(p<.05)$.

$21=E S C B$ (exploded sugar cane bagasse); $2=4 \% \mathrm{Na}_{2} \mathrm{~S}+6 \% \mathrm{NaOH} ; 3=2 \%$ $\mathrm{Na}_{2} \mathrm{~S}+3 \% \mathrm{NaOH} ; 4=9 \% \mathrm{H}_{2} \mathrm{O}_{2}+7 \% \mathrm{NaOH}$.

ração contendo $\mathrm{BAH}$ do presente experimento foram próximos aos atingidos por BERCHIELLI et al. (1989), que registraram valores médios para o CDMS de $45,6 \%$ em rações contendo $\mathrm{BAH}$, fornecidas a bovinos. Todas as dietas contendo os bagaços tratados física e quimicamente apresentaram valores superiores para o coeficiente da digestibilidade da matéria orgânica $(\mathrm{CDMO})$ em relação à dieta à base de $\mathrm{BAH}(\mathrm{P}<0,05)$, de $46,9 \%$, valor este inferior ao obtido por CONCEIÇÃO et al. (1986), que registraram $55,1 \%$ para rações contendo $90 \%$ de BAH na MS fornecida a ovinos. A ração número $2\left(4 \% \mathrm{Na}_{2} \mathrm{~S}\right.$ $+6 \% \mathrm{NaOH}$ ) apresentou o maior $\mathrm{CDMO}$ em relação às demais dietas $(\mathrm{P}<0,05)$, atingindo $66,1 \%$, superior ao valor encontrado por HORTON et al. (1991), de
$45,7 \%$, em rações contendo $60 \%$ de bagaço de cana tratado com pressão e vapor de $20 \mathrm{kgf} / \mathrm{cm}^{2}$ mais $3 \%$ de $\mathrm{NH}_{3}$. Estes resultados observados tanto para a MS como para a MO, particularmente da dieta 2, atestam para a maior disponibilidade dos nutrientes.

A proteína bruta e o extrato etéreo das rações não apresentaram diferenças estatísticas, o que pode ser explicado pelo fato do bagaço ser extremamente pobre nestes nutrientes, e em sua maioria foram provenientes dos alimentos que compuseram o alimento concentrado da ração, igual para todos os tratamentos.

Os valores dos coeficientes de digestibilidade dos extrativos não-nitrogenados (CDENN) das rações à base dos bagaços dos tratamentos 1 (BAH) e 3 $\left(2 \% \mathrm{Na}_{2} \mathrm{~S}+3 \% \mathrm{NaOH}\right)$ não diferiram entre si, mas foram inferiores $(\mathrm{P}<0,05)$ aos valores apresentados pelas dietas contendo os bagaços dos tratamentos 2 $\left(4 \% \mathrm{Na}_{2} \mathrm{~S}+6 \% \mathrm{NaOH}\right)$ e $4\left(9 \% \mathrm{H}_{2} \mathrm{O}_{2}+7 \% \mathrm{NaOH}\right)$.

Todas as rações apresentaram coeficientes de digestibilidade da fibra em detergente neutro (CDFDN) e da fibra em detergente ácido (CDFDA) diferentes entre si $(\mathrm{P}<0,05)$ destacando-se a ração contendo o bagaço tratado com $4 \% \mathrm{Na}_{2} \mathrm{~S}+6 \% \mathrm{NaOH}$, que apresentou o maior valor para os coeficientes das três frações fibrosas. Diferenças observadas nos valores de digestibilidade devem-se a diversos fatores, entre eles as diferentes condições de tratamento do bagaço.

Os CDFDN e CDFDA obtidos por CONCEIÇÃO et al. (1986) para rações contendo BAH foram maiores, de 49,9 e $42,2 \%$, respectivamente, que os obtidos no presente ensaio de 21,1 e $25,5 \%$, respectivamente. Contudo, HORTON et al. (1991), que trabalharam com BAH peletizado com $\mathrm{NH}_{3}$, detectaram valores de CDFDN e CDFDA de apenas 25,2 e $19,9 \%$, respectivamente.

Em suma, a maior digestibilidade registrada para as frações fibrosas FDN e FDA foi para a dieta contendo o bagaço do tratamento $2\left(4 \% \mathrm{Na}_{2} \mathrm{~S}+6 \%\right.$ $\mathrm{NaOH}$ ), sendo seguida por aquela do tratamento 4 $\left(9 \% \mathrm{H}_{2} \mathrm{O}_{2}+7 \% \mathrm{NaOH}\right)$; como o bagaço de cana é constituído primordialmente por parede celular, nada mais lógico que as digestibilidades observadas para a MS e MO seguissem o mesmo padrão das porções fibrosas. O menor teor de lignina em detergente ácido dos bagaços processados pelos tratamentos 2 e 4 , particularmente para o tratamento 2 (Tabela 1), corrobora esta constatação, pois sabe-se que a lignina é um dos principais fatores a inibir a digestibilidade da parede celular das plantas forrageiras (FUKUSHIMA e PANETO, 1995).

Os valores de NDT observados neste trabalho 
seguiram o padrão apresentado pelo CDMS e pelo CDMO, ou seja, a ração contendo o bagaço tratado com $4 \% \mathrm{Na}_{2} \mathrm{~S}+6 \% \mathrm{NaOH}$ (tratamento 2) foi superior àquela contendo o bagaço tratado com $9 \% \mathrm{H}_{2} \mathrm{O}_{2}+$ $7 \% \mathrm{NaOH}$ (tratamento 4) $(\mathrm{P}<0,05)$ e estes dois superiores às dietas dos tratamentos $1(\mathrm{BAH})$ e 3 $\left(2 \% \mathrm{Na}_{2} \mathrm{~S}+3 \% \mathrm{NaOH}\right)(\mathrm{P}<0,05)$, que não diferiram entre si. BERCHIELLI et al. (1989) registraram NDT médio de 51,27\%, para rações contendo BAH, ligeiramente mais elevado que o obtido na presente pesquisa para o mesmo tipo de material. Entretanto, o NDT registrado para as dietas à base dos bagaços processados pelos tratamentos 2 e 4 foram significativamente superiores ao do tratamento BAH $(\mathrm{P}<0,05)$. Pode-se inferir que a superioridade do NDT da ração contendo o bagaço do tratamento 2 é reflexo dos melhores índices de digestibilidade dos nutrientes, particularmente das frações fibrosas do bagaço deste tratamento, em relação às demais rações.

Embora os ensaios de digestibilidade in vitro tenham sido realizados apenas com os bagaços tratados e os experimentos de digestibilidade in vivo, com dietas completas, podem-se observar menores valores de digestibilidade in vivo em relação ao ensaio de digestibilidade in vitro, tanto para a MS como para a MO, à exceção do BAH. A explicação mais evidente foi apresentada por BERGER et al. (1979), os quais relataram que a elevada taxa de passagem ruminal, devido às altas concentrações de substâncias minerais dos bagaços tratados quimicamente, que acarreta maior ingestão de água, diminui o tempo de retenção do alimento no rúmen, diminuindo, portanto, o tempo de contato do substrato com as enzimas microbianas. Corrobora esta tese a observação de que o único bagaço (ração) a não exibir menor valor de digestibilidade in vivo em relação ao seu similar da digestibilidade in vitro foi o $\mathrm{BAH}$, ou seja, aquele bagaço não tratado quimicamente.

\section{Conclusões}

Emprego de reagentes químicos e uso de tratamento físico incrementa a digestibilidade do bagaço de cana, particularmente das frações fibrosas, o que resulta em maior valor de NDT, permitindo, dessa maneira, menor participação de alimento concentrado no arraçoamento animal. Constata-se, por conseguinte, o excelente potencial de aproveitamento deste resíduo da agro-indústria sucro-alcooleira para fins de alimentação de ruminantes.

\section{Agradecimento}

Ao pessoal docente e técnico do Departamento de Ciências Florestais da Escola Superior de Agricultura "Luiz de Queiroz" da Universidade de São Paulo, por terem permitido acesso e uso de seus equipamentos, e também aos técnicos e funcionários da Usina Santa Luíza (Matão, SP), por, gentilmente, terem doado o bagaço e permitido a utilização do equipamento de hidrólise.

À FAPESP, pelo auxílio financeiro ao projeto e pelas bolsas de Mestrado e de Iniciação Científica.

\section{Referências Bibliográficas}

AMJED, M., JUNG, H.G., DONKER, J.D. 1992 Effect of alkaline hydrogen peroxide treatment on cell wall composition and digestion kinects of sugar cane residues and wheat straw. J. Anim. Sci., 70:2877-2884.

ANDRADE, M.B.M., FUKUSHIMA, R.S. Efeitos de diferentes tratamentos no bagaço de cana sobre a digestibilidade dos nutrientes. I. Digestibilidade “in vitro". In: REUNIÃO ANUAL DA SOCIEDADE BRASILEIRA DE ZOOTECNIA, 31, 1994, Maringá. Anais... Maringá: SBZ, 1994, p.514.

ASSOCIATION OF OFFICIAL ANALYTICAL CHEMISTS AOAC. 1990. Official methods of analysis. 15.ed. Washington D.C. $1298 \mathrm{p}$.

ASSUMPÇÃO, R.M.V. 1988. Polpação química. In: D'ALMEIDA, M.L.O. Celulose e papel: tecnologia de fabricação da pasta celulósica. São Paulo: SENAI/IPT, v.1, 2.ed, p. 169-319.

BEM, C.H.W. Efeito de bicarbonato de sódio elou lasalocida sobre digestibilidade de dietas com bagaço de cana. Piracicaba, SP: ESALQ, 1991.71p. Dissertação (Mestrado em Zootecnia) - Escola Superior de Agricultura "Luiz de Queiroz"/ Universidade de São Paulo, 1991.

BERCHIELLI, T.T., ANDRADE, P., RIBEIRO, M.V. et al. 1989. Digestibilidade de rações contendo bagaço de cana hidrolisado, suplementadas com farelo de algodão, levedura e rolão de milho. Rev. Soc. Bras. Zootec., 18(6):532-537.

BERGER, L., KLOPFENSTEIN, T., BRITTON, R. 1979. Effects of sodium hydroxide on efficiency of rumen digestion. J. Anim. Sci., 49:1317-1327.

BOIN, C., MATTOS, W.R.S., D’ARCE, R. 1987. Cana-deaçúcar e seus sub-produtos na alimentação de ruminantes. In: Cana-de açúcar: cultivo e utilização. São Paulo: Fundação Cargill, v.2, p.805-856.

BURGI, R. Produção do bagaço de cana-de-açúcar (Saccharum sp L.) auto-hidrolisado e avaliação para ruminantes. Piracicaba, SP: ESALQ, 1985.61p. Dissertação (Mestrado em Zootecnia) - Escola Superior de Agricultura "Luiz de Queiroz"/ Universidade de São Paulo, 1985.

CASTRO, F.B., MACHADO, P.F. 1989. Avaliação do processo de digestão do bagaço de cana-de-açúcar tratado sob pressão e vapor. Bol. Ind. Anim., 46:213-217.

CHEONG, Y.W.Y., D'ESPAIGNET, J.T., DEVILLE, P.J. The effects of steam treatment on cane bagasse in relation to its digestibility and furfural production. In: CONGRESS INTERNATIONAL SOCIETY OF SUGAR CANE TECHNOLOGISTICS, 1974, Mauritius. Proceedings... Mauritius: ISSCT, 1974, p.1-18. 
1204 Rev. bras. zootec.

CONCEIÇÃO, M.N., LACÔRTE, M.C.F., BURGI, R. et al. Determinação da digestibilidade do bagaço de cana-de-açúcar hidrolisado com carneiros. In: REUNIÃO ANUAL DA SOCIEDADE BRASILEIRA DE ZOOTECNIA, 23, 1986, Campo Grande. Anais... Campo Grande: SBZ, 1986, p. 156.

DANILAS, R.M. 1988. Branqueamento de pastas celulósicas. In: D'ALMEIDA, M.L.O. Celulose e papel: tecnologia de fabricação da pasta celulósica. São Paulo: SENAI/IPT, v.1, 2.ed, p.427-512.

FAHEY JR., G.C., BOURQUIN, L.D., TITGEMEYER, E.C. et al. 1993. Postharvest treatment of fibrous feedstuffs to improve their nutritive value. In: JUNG, H.G, BUXTON, D.R., HATFIELD, R.D., RALPH, J. (Eds.) Forage cell wall structure and digestibility. Madison: ASA - CSSA - SSSA. p.715-766.

FUKUSHIMA, R.S., PANETO, J.C.C. 1995. A lignina e o valor nutritivo das plantas forrageiras: uma revisão. Zootecnia, 33:29-39.

FUKUSHIMA, R.S., DEHORITY, B.A., LOERCH, S.C. 1991. Modification of a colorimetric analysis for lignin and its use in studying the inhibitory effect of lignin on forage digestion by rumen microorganisms. J. Anim. Sci., 69:295.

GOERING, H.K., VAN SOEST, P.J. 1970. Forage fiber analysis (apparatus, reagents, procedures and some applications). Washington, D.C.: USDA, Agricultural Handbook n. 379. 20p.

HART, M.R., WALKER JR., H.G., GRAHAM, R.P. et al. 1981. Steam treatment of crop residues for increased ruminant digestibility. I. Effects of process parameters. J. Anim. Sci., 51:402-413.

HORTON, G.M.J., PATE, F.M., PITMAN, W.D. 1991. The effects of steam-pressure treatment, pelleting and ammoniation on the feeding value of sugar cane bagasse for cattle. Can. J. Anim. Sci., 71:79-86.

JACKSON, M.G. 1977. The alkali treatment of straws. Anim. Feed Sci. Technol., 2:105-130.

MARCOS, A.C.M., LEME, P.R., BOIN, C. 1984. Efeito do tempo de tratamento a pressão de vapor na composição química e na digestibilidade in vitro da matéria seca do bagaço de cana-de-açúcar. Zootecnia, 22:383-395.

MARTIN, P.C., CABELlO, A., ELIAS, A. 1976. The use of fibrous sugar cane by products by ruminants. 2. Effects of $\mathrm{NaOH}$ pressure combination on the digestibility and chemical composition of bagasse and bagasse pith. Cuban J. Agric. Sci., 10:19-28.
NATIONAL RESEARCHCOUNCIL - NRC. 1985. Requirements of sheep. 6.ed. Washington D.C., National Academy of Science. 99p.

NOLTE, M.E., CLINE, J.H., DEHORITY, B.A. et al. 1987. Treatment of wheat straw with alkaline solutions prepared prepared from wood ashes to improve fiber utilization by ruminants. J. Anim. Sci., 64:669-677.

NUSSIO, L.G. Efeitos de níveis de concentrado sobre o desempenho de bovinos e digestibilidade de dietas a base de bagaço de cana-de-açúcar (Saccharum $s p$ L.) tratado sob pressão de vapor. Piracicaba, SP: ESALQ, 1993. 147p. Dissertação (Mestrado em Zootecnia) - Escola Superior de Agricultura "Luiz de Queiroz"/Universidade de São Paulo, 1993.

RANGNEKAR, D.V., BADVE, V.C., KHARAT, S.T. et al. 1982. Effect of high-pressure steam treatment on chemical composition and digestibility in vitro of roughages. Anim. Feed Sci. Technol., 7:61-70.

REXEN, P.F. 1979. Low quality forages improved with alkali treatment. Feedstuffs, 51:33-34.

STEEL, G.D., TORRIE, J.H. 1980. Principles and procedures of statistics: a biometrical approach. 2.ed. New York: McGraw - Hill Book. 633p.

VAN SOEST, P.J. 1963. Use of detergents in the analysis of fibrous foods. II. A rapid method for the determination of fibre and lignin. J. Assoc. Off. Anal. Chem., 46:829.

VAN SOEST, P.J. 1994. Nutritional ecology of the ruminant. 2.ed. Ithaca: Comstock Publishing Associates. 476p.

Recebido em: 06/11/1998

Aceito em: 28/12/1999 